\title{
Predicting moisture content of chipped pine samples with a multi-electrode capacitance sensor
}

Pengmin Pan ${ }^{a}$, Timothy McDonald ${ }^{\mathrm{b}}$, Brian Via ${ }^{\mathrm{c}}$, John Fulton ${ }^{\mathrm{d}}$, \& John Y. Hung ${ }^{\mathrm{e}}$

${ }^{a}$ Department of Biosystems Engineering, Auburn University, AL, USA. Address: 308 Corley Building, Auburn, AL 36849, USA. Tel: (334) 844-3550, Email: pzp0014@auburn.edu ${ }^{\text {b }}$ Corresponding author. Department of Biosystems Engineering, Auburn University, AL, USA. Address: 224 Corley Building, Auburn, AL 36849, USA. Tel: (334) 844-3545, Email: mcdontp@auburn.edu

c School of Forestry and Wildlife Sciences, Auburn University, AL, USA. Address: 520 Devall Drive, Auburn, AL 36849, USA. Tel: (334) 844-1088, Email: bkv0003@auburn.edu ${ }^{\text {d }}$ Department of Food, Agricultural and Biological Engineering, The Ohio State University, OH, USA. Address: 212 Agricultural Engineering, 590 Woody Hayes Drive, Columbus, OH 43210, USA. Tel: (614) 292-6625, Email: fulton.20@osu.edu ${ }^{\mathrm{e}}$ Department of Electrical \& Computer Engineering, Auburn University, AL, USA. Address: 200 Broun Hall, Auburn, AL 36849, USA. Tel: (334) 844-1813, Email: hungjoh@auburn.edu

Nomenclature

$\alpha, \beta$, and $k$ : Model coefficients (constants)

$\epsilon$ : Random error

$C$ : Sensor output (V)

G: Permittivity distribution matrix

$S$ : Sensitivity matrix

M: Mass (g)

ECT: Electrical capacitance tomography

MC: Moisture content

NIR: Near infrared

SPP: Single paired parallel-plate

RMSE: Root mean square error 


\section{INTRODUCTION}

In the southern USA and elsewhere woody biomass is most often sold as pulpwood and sawn logs on a wet mass basis, a system that works because the moisture content (MC) of harvested trees normally fluctuates within a narrow range and wood from multiple sources can be considered a uniform product. However, when MC varies outside its typical range, the use of wet mass as a basis for selling timber products becomes untenable. This is particularly true in energy markets, where moisture is often considered a contaminant and there are methods of altering MC of feedstocks before shipment. A moisture sensor that could be widely accepted and could work reliably and accurately across a broad range of product forms and MC would help in promoting robust and stable biomass for energy markets. Ideally, such a sensor would be useful not only in laboratory settings when evaluating relatively small samples of material, but also in applications in where the material to be assessed was flowing and when the quantity to be evaluated was very large.

Multiple methods and technologies have been investigated to evaluate MC of biomass, including dielectric property measurements (James, 1963; Kandala \& Sundaram, 2010; Kandala \& Puppala, 2012), X-ray (Roels \& Carmeliet, 2006), microwave (Johansson, 2001; Vallejos \& Grote, 2009; Hansson et al., 2005; Trabelsi et al., 2013), acoustic sensing (Minamisawa et al., 1990; Amoodeh et al., 2006), radio-frequency scanning (Hanson \& Kelly, 1998; Barale et al., 2002) and near infrared spectroscopy (NIR) (Mesic et al., 2005; Adedipe \& Dawson-Andoh, 2008; Jensen et al., 2006; Defo et al., 2007; Tormanen \& Makynen, 2011). Of the available technologies, the most likely to be successful for both bulk static and flowing materials are NIR and dielectric sensing (Nyström \& Dahlquist, 2004). Both these technologies have been shown effective in measuring MC of diverse biological materials. NIR systems have been developed, for example, for analysing wood chips (Axrup et al., 2000), forage (Digman \& Shinners, 2008) and other biomass (such as Mesic et al., 2005).

Dielectric methods are used to measure permittivity of woody biomass, which is known to correlate with MC (Nelson, 1991). One of the most common dielectric transducers used in assessing permittivity is a capacitance sensor (Kandala \& Sundaram, 2010). It is a simple and robust system that can independently measure mass and MC of biomass (Kandala \& Sundaram, 2010; Kandala \& Puppala, 2012). However, when the distribution of MC within the sample is not uniform, the single capacitance sensor cannot provide any information about the sample's variability. The use of multiple electrodes provides the opportunity to probe the distribution of MC in a bulk sample using a tomographic approach (Jazayeri \& Ahmet 2000). This technology is referred to as electrical capacitance tomography (ECT). Sensors having eight (Huang et al., 1988), twelve (Olmos et al., 2007) or sixteen (Wang et al., 2009) electrodes have been developed. ECT has been widely applied in, for example, (1) evaluating distributions of multiple-phased flow in pipes (two phase flow: Xie et al., 2007; Li et al., 2008; Johana et al., 2011; three phase flow: Li et al., 2012), (2) measuring mass flow rate of solid materials (Young et al., 1996; Sun et al., 2008), and (3) sensing the distribution and volume of water inside mixtures of sand and clay (Karim \& Ismail 2011).

The research reported here was aimed at evaluating the application of ECT for the accurate, rapid, noncontact measurement of MC in biomass (specifically, pine chip) flows. However, preceding that aim it was necessary to determine how well the system functioned in a static bulk measurement mode. This paper, therefore, presents details of the ECT sensor development and described its accuracy relative to a widely accepted method in bulk biomass MC measurements, NIR. The experiments conducted were designed to evaluate both sensing technologies under the best possible conditions for each. Because it is essentially a surface measurement, it was hypothesised that NIR would be best applied in predicting MC for situations where the dry density and moisture distribution of the material under test could be considered uniform. The first objective in this study was, therefore, to compare NIR and ECT MC measurements on the smallest, most uniform volume of material available, for this study an individual wood chip. ECT, on the other hand, interacts with a volume of material equivalent to the interior dimensions of the sensor itself and provides a true bulk measurement. In this study, the sensor volume was constructed to measure MC of about $1000 \mathrm{~cm}^{3}$ of biomass at a time and the second objective was to compare MC estimates for quantities of biomass of 
that size. It was hypothesized MC estimates from ECT for a larger volume of biomass having non-uniform physical properties would be more accurate than those from NIR.

\section{Sensor Development and Validation}

\subsection{ECT Sensor Specifics}

An 8-electrode ECT sensor was designed to determine the MC of loblolly pine pulpwood chips. A 152-mm diameter PVC pipe was selected as the measuring vessel. Electrodes formed from copper plates $100.0 \mathrm{~mm}$ $(\mathrm{L}) \times 25.4 \mathrm{~mm}(\mathrm{~W})$ attached using an adhesive uniformly around the perimeter of the pipe. The plates were used in pairs to generate an electric field within the pipe, the strength of which was related to the permittivity of the material between the electrodes. The pairs of electrodes were viewed as a single capacitive element in a circuit generating an electric field. The capacitance of the electrode pairs was related to the permittivity of the material filling the void between them. Application of the ECT sensor therefore involved the sequential measurement of capacitance between each unique pair of electrodes. The specifics of the sensing circuit used to measure capacitance were adapted from Yang \& York (1999). There were two main elements of the system (Fig. 1), the first of which was a filter having a gain proportional to the capacitance of the electrode pairs. The filter was constructed using Analog Devices AD844 op-amps (Analog Devices, Inc., Norwood, MA, USA). The second element of the sensing system was a switching network used to connect each electrode individually into the filter circuit.

The capacitances to be measured between any pair of electrodes were very small and variations caused by the tested material were even smaller. Any non-constant stray capacitance arising from an internal or external source would degrade the sensitivity of the sensor and affect accuracy. Stray capacitances were introduced primarily from the switching network used to connect the electrodes into the sensor circuit. Each electrode had to be coupled to the input signal source, to the input of the filter circuit, or remain disconnected and this required at least one three-state switch. When implemented in the sensor circuitry, however, switches for the six electrodes in the off state created a strong stray capacitance and interfered significantly with capacitance measurements. We therefore chose to apply three Analog Devices ADG419 (Analog Devices, Inc., Norwood, MA, USA) two-pole switch devices for each electrode, one to connect it to either the source or filter circuits, plus another to shunt the electrode to ground when it was not actively involved in the measurements. The ADG419 devices were chosen based on their very low off-state leakage currents. Switch leakage currents effectively introduced a parallel capacitance to ground that, if the current was large enough, it would overwhelm that due to the dielectric material in the sensor.

The filter circuit shown in Fig. 1 functioned as a capacitance-to-voltage converter and its output was a scaled version of its input, in this case a $800 \mathrm{kHz}$ sine wave. The amplitude of the filter's output sine wave was, therefore, proportional to the capacitance of the electrode/biomass combination. A fast Fourier transform (FFT) approach was used to extract the filter output amplitude. The method involved transforming a 1024-point sequence of the output sampled at $2 \mathrm{MHz}$ and recording the FFT response at the frequency closest to $800 \mathrm{kHz}$. These data were acquired using a NI6361 multifunction I/O device (National Instruments Co., Austin, TX, USA) and processed using LABVIEW ${ }^{\mathrm{TM}} 2013$ (National Instruments Co., Austin, TX, USA) software.

\subsection{Sensor Characterisation}

Preliminary tests were carried out to investigate the response of the ECT sensor to MC in pine chips in its two sensing modes: single paired parallel-plate (SPP) mode, and tomographic. Of particular concern in this analysis was to understand the response of the sensor to a heterogeneous material containing both wood and water bound together. Individually, the dielectric constants (at room temperature) of air $\left(\varepsilon_{r}=1\right)$, dry wood (3-5), and water (80) are quite different (Norimoto, 1976) and the sensor should have been able to discriminate them. It was not known, however, how the sensor would behave with those three constituents combined. 


\subsubsection{Single Paired Parallel-Plate Test Mode}

The SPP mode application of the sensor was intended for use as a means of sensing MC of a bulk material under static conditions. It was envisioned the sensor would be filled with some known quantity of material (to account for the mass effect), a series of individual capacitance measurements using the sensor's multiple electrodes made and combined, and the result would be a good predictor of MC.

Two different tests were conducted to investigate the sensor response to blended materials. The first involved placing different quantities of dry wood into the sensor and generating a reading. The second test used a fixed quantity of chips of different MC. Pine chips plus water were repeatedly dried, weighed, and then measured using the ECT sensor over the entire expected range of MC.

Each SPP mode sensor measurement for a single biomass sample consisted of the average of five sequential observations of the summed capacitance from each of the 28 unique electrode combinations. The response was calculated relative to a corresponding background estimate derived from the empty sensor. Chip samples were obtained from loblolly pine obtained from a local mill. The results of the two experiments indicated a linear response to both constituents (wood and water, Fig. 2) and implied that the two effects (water and wood mass) could be separated based on sensor readings, given some additional, independent measure of one or the other.

The MC, therefore, could be modelled using two linear effects as in Eq (1).

$$
\Delta C_{\text {sum }}=\alpha M_{\text {water }}+\beta M_{d r y}+\epsilon
$$

The $\Delta C_{\text {sum }}$ term was the cumulative sensor output (relative to empty), $M_{\text {water }}$ and $M_{d r y}$ were masses of water and dry wood, respectively, $\alpha$ and $\beta$ were sensor gains associated with water and dry wood, respectively, and $\epsilon$ was a normally distributed random error. If we define $k=\beta-\alpha$, for the wet mass of a sample: $M_{\text {wet }}=M_{d r y}+M_{\text {water }}$, Eq (1) can be rewritten as:

$$
\Delta C_{\text {sum }}=\alpha M_{\text {wet }}+k M_{d r y}+\epsilon
$$

The model coefficients $\alpha, \beta$, and $k$ could be estimated experimentally, and the dry-basis MC could then be calculated based on sensor output and wet mass as:

$$
M C=\frac{k M_{\text {wet }}}{\Delta C_{\text {sum }}-\alpha M_{\text {wet }}}-1
$$

Calculating MC of a sample, therefore, was based on its wet mass, the sensor output, and previously defined regression coefficients.

\subsubsection{Tomographic Mode}

The ability to image MC variations of biomass was an important motivating factor in developing the ECT sensor and the procedures for its application were developed and tested in this study. Calculating the twodimensional permittivity distribution was based on the Linear Back Projection (LBP) method (Xie et al., 1990), which transformed a set of measured parallel-plate capacitances, $C$, into an array of node permittivities $G$ at fixed locations within the sensor enclosure using the relation $C=S G$. The matrix $S$, or sensitivity matrix, represented the normalised variation in permittivity at the same node locations with some reference material filling the enclosure. It was generated using simulations of the electric field created within the sensor for all the unique combinations of two electrodes, one plate held at ground potential, the other with fixed excitation. Finite element simulations to calculate $S$ were carried out using the Ansys ${ }^{\circledR} 9.0$ software package (Ansys Inc., Cecil Township, PA, USA). Higher-resolution images of the permittivity 
distribution (as in Fig. 3 below) were derived from interpolated $G$ values and were calculated using the scipy.interpolate package in Python (Python Software Foundation, Wilmington, DE, USA) with a radial basis function.

The ability of the ECT sensor to image the radial distribution of MC in chip samples was tested using three different spatial arrangements of dry (approximately 5\%, d.b.) and wet (65\%, d.b.) wood chips. The first test stacked three regions across the width of the sensor enclosure with regions contained $200 \mathrm{~g}$ dry and $200 \mathrm{~g}$ of wet chips separated by an air gap. In the second region, $200 \mathrm{~g}$ dry chips were placed around the perimeter of the sensor surrounding $200 \mathrm{~g}$ wet chips in the middle. In the final arrangement a mixture of $200 \mathrm{~g}$ dry and $200 \mathrm{~g}$ wet chips was simply poured into the sensor.

Ten repeated ECT readings were collected for each sample arrangement. An ANOVA test was performed with arrangement as the independent variable and null hypothesis that SPP-mode MC readings for all samples were the same. Results showed no significant difference among observed means $(P=0.76)$, suggesting the ECT sensor output was robust to the physical arrangement of the sample material within the measurement enclosure. Figure 3 shows cross-sectional images of all sample arrangements and illustrated the ability of the ECT system to effectively discriminate the 2-dimensional distribution of permittivity in pine chips. This confirmed the unique aspect of ECT that cannot be achieved using a single paired electrode capacitance sensor.

\section{Method and Materials}

Samples for assessing the difference between NIR and capacitance MC assessment methods were obtained from six batches of loblolly pine pulpwood chips collected from three local sources. Four of the batches were fresh and green (Batches 1-4), two of the batches had been stored for about six months, one was piled green (Batch 6) and the other had been air-dried in a thin layer for about two months (Batch 5).

For the individual chip test, samples from one of the fresh batches (Batch 1) were used for calibration of models and samples from it and the remaining five were used for validation. The ECT sensor as used in this study could not reliably detect quantities of wood less than about $10 \mathrm{~g}$ dry mass, so batches were screened to separate larger chips and the ones used in the model calibration and validation were randomly selected from among the remaining larger specimens. Individual chips were dried in a $105^{\circ} \mathrm{C}$ oven for varying lengths of time to develop a range of $\mathrm{MC}$ among the samples. After drying, chips were assessed using the NIR system followed immediately by ECT measurements on the same samples. Calibrations between sensor outputs and MC were built using 50 individual chips and validated using another 34 .

Measurements on bulk samples were made on subsamples of 40-80 chips from single batches. The chips were assembled into groups from the quantity available and each group sample was weighed and then dried for a varying period of time, as in the individual chip tests. This process resulted in bulk samples ranging in average MC from "near bone dry" to about $110 \%$ (d.b.). A total of 30 groups was used for calibration and 18 for validation.

\subsection{NIR Measurements}

A PerkinElmer Spectrum 100N FT-NIR spectrometer (Perkin-Elmer Inc., Waltham, MA, USA) equipped with a fibre-optic probe was used to collect wood chip spectra. The wavelength range of the spectrometer was from $1000 \mathrm{~nm}$ to $2439 \mathrm{~nm}$, which covers two major absorption bands of water.

For each chip tested in the individual chip measurement comparison, NIR spectra were taken from two randomly selected points, one on each large side. Each spectrum was an average of 16 samples taken consecutively. The software Spectrum Quant+ 4.6.0 (Perkin-Elmer Inc., Waltham, MA, USA) was used to baseline correct and smooth the spectra. The final spectrum used to represent a single chip was the average of those processed spectra taken from both sides. 
Principle component analysis (PCA) was used to extract linearly independent variables (principle components, PCs) from spectral data. PCs were chosen to build the calibration model linking measured spectra to MC using the multiple linear regression method (Via et al., 2003).

For bulk measurements, NIR spectra of all individual chips comprising the sample were measured in the same manner as in the single chip tests. Calibration models predicting average MC of the entire bulk sample were built using random subsets of the capacitance data with increasing proportions of the values from individual chips included in the assessment. Validation tests were done using the same approach, also employing increasing proportions of the sample in the measurement set representing the bulk characteristic.

\subsection{ECT Measurements}

ECT measurements for individual chips were made with the chip placed on a PVC support located near the middle of the space surrounded by sensor electrodes. Bulk measurements were made with the sample simply poured into the sensor enclosure to a uniform depth. A single measurement consisted of the sum of all unique combinations of sensor outputs from electrode pairs with the sample in place minus the observed output for the pair without the sample in place.

\section{Results}

Root mean square error (RMSE) was used to describe the differences between estimated and measured quantities. Given a true (measured) value $y_{n}$ and a predicted value $\widehat{y_{n}}$, the RMSE of the prediction (RMSEP) is defined as in Eq (4).

$$
R M S E=\sqrt{\frac{\sum_{i=1}^{n}\left(\widehat{y_{n}}-y_{n}\right)^{2}}{n}}
$$

\subsection{Individual Chips}

Predictions of MC of individual chips were generally more accurate using NIR than ECT sensors. Figure 4 shows the measured and estimated MCs, Fig. 4a for NIR and Fig. 4b for ECT. Each plot includes the calibration set data (from a single batch), plus predicted results for chips from all five batches. The coefficients of determination shown were for the combined data (calibration plus prediction). Table 1 lists standard errors for the calibration and prediction sets by sensor and sampling method (individual or bulk). The overall prediction errors for individual chips using the ECT sensor were about 2.5 times that of NIR and were over four times larger for the chips that had been stored outdoors for six months (batch 6). These chips were less homogenous in nature than fresh batches (larger amounts of dirt and contaminants) and also tended to be very wet, typically wet to the touch. Their MC was outside the range tested in the calibration set and was also outside the predicting capability of the calibration model.

The relatively poor results from ECT were likely due to the sensor operating near its detection threshold for samples as small in mass as a single chip. Such small quantities of material in the sensor produced output signals only marginally larger than background noise (SNR $<4 \mathrm{~dB}$ ). This result indicated the difficulty in designing an ECT sensor to work efficiently across a broad range of sample quantity for materials consisting of two distinct phases (wood and water, in this case) because of its mass dependency.

\subsection{Bulk Measurements}

ECT measurements of MC on bulk samples of chips were generally accurate, with mean RMSEP equal to $11.65 \%$ (Table 1) based on a calibration set from a single batch of chips (Batch 1). The RMSEP values for single batches were less than $8 \%$ in all cases except batch 6 , which was, as in the individual chip results, 
more than twice as large. Again, however, batch 6 MCs were outside the range of calibration and this fact may have helped explain the poor performance of the ECT system for that material.

Figure 5 shows the performance of NIR and ECT predictions of MC for bulk samples. The NIR response shown in the figure resulted from a $100 \%$ sampling of the chips in the group, i.e., spectra were collected from all chips in the bulk sample and then averaged. Results of the NIR testing with $100 \%$ sampling were comparable to ECT predictions, but were slightly better for ECT across all samples and for those in specific batches (Table 1).

Using less than $100 \%$ of the individual chips in the NIR measurements to calibrate the bulk MC model resulted, as one would expect, in a greater amount of variability in the validation samples. Table 2 shows RMSEP values when spectra from a varying percentage of chips in the calibration set samples were used to develop the model. Chips were randomly selected from each bulk sample for inclusion in both the calibration and validation data sets. Errors increased when fewer chips from a bulk sample were used in calibrations, and using a $40 \%$ sampling rate more than doubled the RMSEP relative to $100 \%$ sampling for most batches.

\section{Discussion}

For predicting the MC of small quantities of biomass, for amounts that could be considered to have uniform MC the NIR method was superior. With more spectral averaging per single chip (multiple spectra collection points per side, for example), the difference in performance relative to ECT could have been even greater. ECT responded to cumulative changes in dielectric, mostly an effect of the total mass present, and this characteristic limited accuracy in tests on individual chips where changes in sensor output were near the minimum resolution of the instrument.

Both methods suffered when samples were not clean, as seen in the results for Batch 6 (Karim \& Ismail, 2011). Batch 6 chips often had significant amounts of free water and soil present on their surface, the result of heavy rains just prior to sample collection. These contaminants were difficult to account for in the calibration process used and prediction errors were several times greater than observed in other batches for both sensors. In NIR measurements the contamination errors could have been compounded by variations in surface roughness, or the proportions of early and late wood sampled at a particular spot. Both of those variations might have been compensated for somewhat by greater sampling density over the surface of the chip. ECT predictions of MC in individual chips should have been less sensitive to the presence of surface moisture because the sensor should have responded to the total mass of water and dry wood under test. There could also have been some additional variation in Batch 6 measurements because of the different amounts of free and bound water between it and the other samples, although the literature (Boyarskii et al., 2002) indicates that this effect should have been small.

NIR predictions of bulk samples composed of individual chips having variable mass and MCs would be expected to have higher errors unless these factors were controlled. In this study, spectrum estimates from each chip included in the bulk sample were combined without regard to chip dry mass and, without compensating for that combined mass/moisture variation, the NIR sensor errors were greater than those observed with ECT. The ECT predictions, on the other hand, should have been immune to variation in individual chip mass because the sensor responded to the cumulative quantities of water and dry wood rather than an estimate of their relative proportions, as in NIR.

Both sensors should be useful in situations where MC of larger populations of chipped, or otherwise comminuted, biomass was predicted using sub-sampling. The ECT approach, however, would be simpler since it should more reliably predict MC on a bulk sample, rather than necessitating the combined size/MC variation in the material being quantified as is required with NIR. Given a relatively uniform distribution in those parameters, however, NIR should be feasible if results from averaged measurements on smaller quantities were sufficient. The ECT sensor had the additional advantage of being a simple and rapid 
measurement technique. With the ECT system used in this study, less than 1 second per reading was required to make a MC determination regardless of sample quantity up to the amount that could be placed within the sensor enclosure.

As used in this study, the ECT sensor required an independent measure of mass to predict MC but there are at least two other practical methods that could be applied using the system to establish mass free moisture prediction models. One would be filling the sensing volume with the sample being investigated. If the population of wood chips under measurement had relatively uniform particle size, the variation of dry mass within the enclosure should vary only within in a narrow range and ECT readings would mainly reflect changes in MC. Another approach would be to use a dual frequency excitation as described by Kandala \& Puppala (2012). The difference in capacitance readings observed between the two frequencies should be only related to MC. Both of these methods were tested for this research using the same methods as with bulk measurements described above. That is using samples from batch 1 for calibration and batches 2-5 for validation. Calibration results were shown in Fig. 6. RMSE of calibration set (RMSEC), RMSEP of the filling and dual-frequency methods were $12.57 \%, 13.45 \%$ and $10.66 \%, 10.93 \%$, respectively. The dualfrequency method achieved a lower variance in its estimates, but required doubled measuring time. However, the method has the potential to be applied easily in moving biomass using two sets of capacitance sensors. The accuracies of both methods were comparable to the mass-involved models above.

Based on the results found in this study, recommendations for measuring MC of batch pine chip samples would be as follows. For very small quantities (e.g. less than 10 individual chips), use NIR; for medium quantities (bulk samples of chips, the volume of which is less than the ECT sensing volume), use ECT and the mass-included model; for a large quantity (i.e. exceeding the ECT sensor measuring volume), use mass free models with two options - faster (fixed volume approach), or more accurate (dual-frequency).

Both sensors should also have utility in on-line measurement of MC in flow applications, but both have limitations as well. For NIR, simultaneous variation in chip size and MC would limit its accuracy in situations where this effect could not be averaged out over time. ECT measurement accuracy would also be limited unless simultaneous estimates of mass flow were available. The imaging capabilities of the system may provide an acceptable estimate of mass flow, but the accuracy of the method was not verified in this study. The ECT combined mass/physical property measurement approach has been tested by others (Arko et al., 1999; Sun et al., 2008) on different multi-phase materials and found to be feasible. Given the accuracy of mass flow measurements based on image reconstruction techniques could be verified, the ECT sensor should be a feasible way to characterize multi-phase biomass and water flow streams as long as frame rates of the imaging system are sufficiently high to capture variability (on the order of 100 frames s${ }^{-1}$, Xie et al., 1992). Even if the imaging approach is not effective in calculating mass flow, it might be predictable using an additional set of electrodes (Arko et al., 1999). Additional information might also be available through measurements of phase angle and impedance variations that have been reportedly used as a means to compensate for errors caused by air gaps (Kandala \& Puppala, 2012). Adding these measures would, however, significantly complicate the sensor electronics.

\section{Conclusions}

NIR methods worked well in predicting moisture for biomass of low quantity and/or samples with uniform properties. However, due to its surface and spot scanning properties, the accuracy could be greatly reduced if measurements were being made on bulk samples, or samples with non-uniform within-chip moisture distribution. Comparatively, the ECT method used was robust to the distribution of biomass with a large moisture gradient. Additionally, the measuring speed of ECT, which was independent of sample quantity (up to the volume of measuring enclosure), was much faster than NIR for bulk samples. However, MC of small samples (e.g. mass of individual chips $<10.0 \mathrm{~g}$ ) was not accurately determined using ECT because of the threshold sensitivity of the system. A reasonable way of using ECT for static MC prediction was to fully fill the sensing area with chipped samples, which could approximate the dry weight of biomass as a constant. The sensor output, in that case, was only related to MC. 
Fig. 1

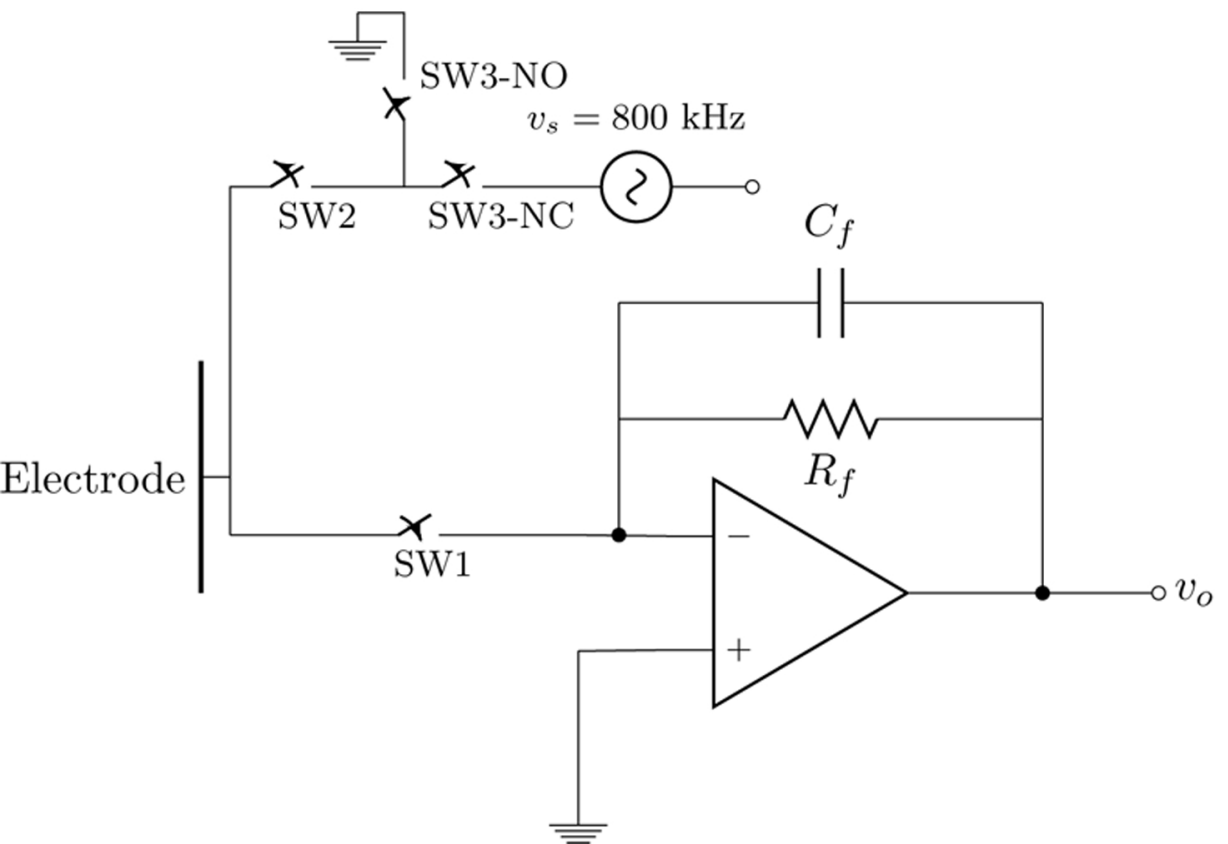




\section{Fig. 2}

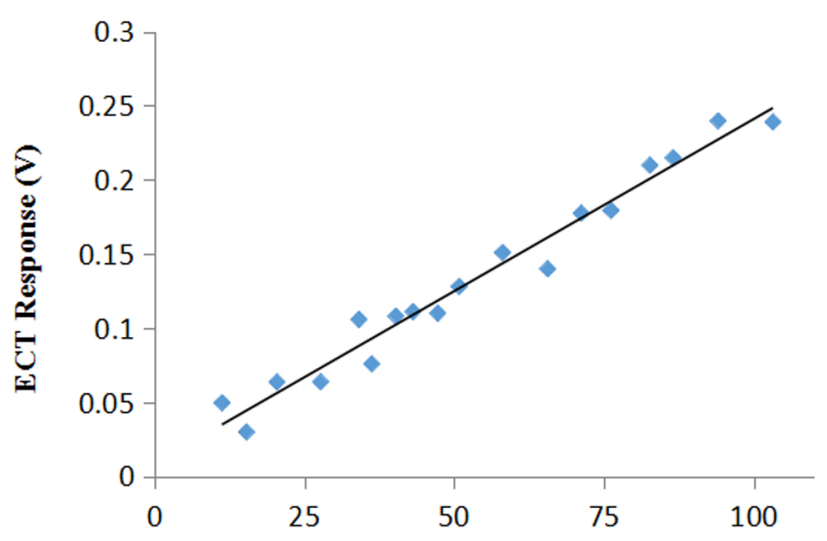

Mass of dry wood chips $\left(M_{d y}\right)(g)$

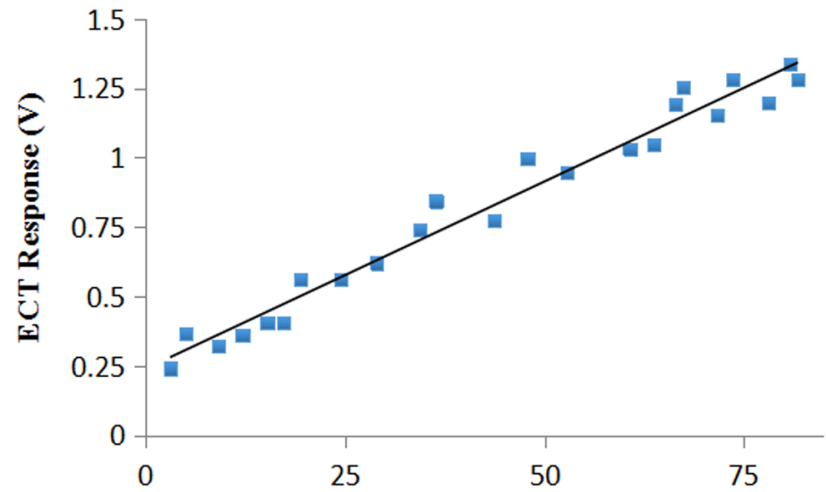

Mass of water in wood chips $\left(M_{\text {water }}\right)(\mathrm{g})$ 
Fig. 3
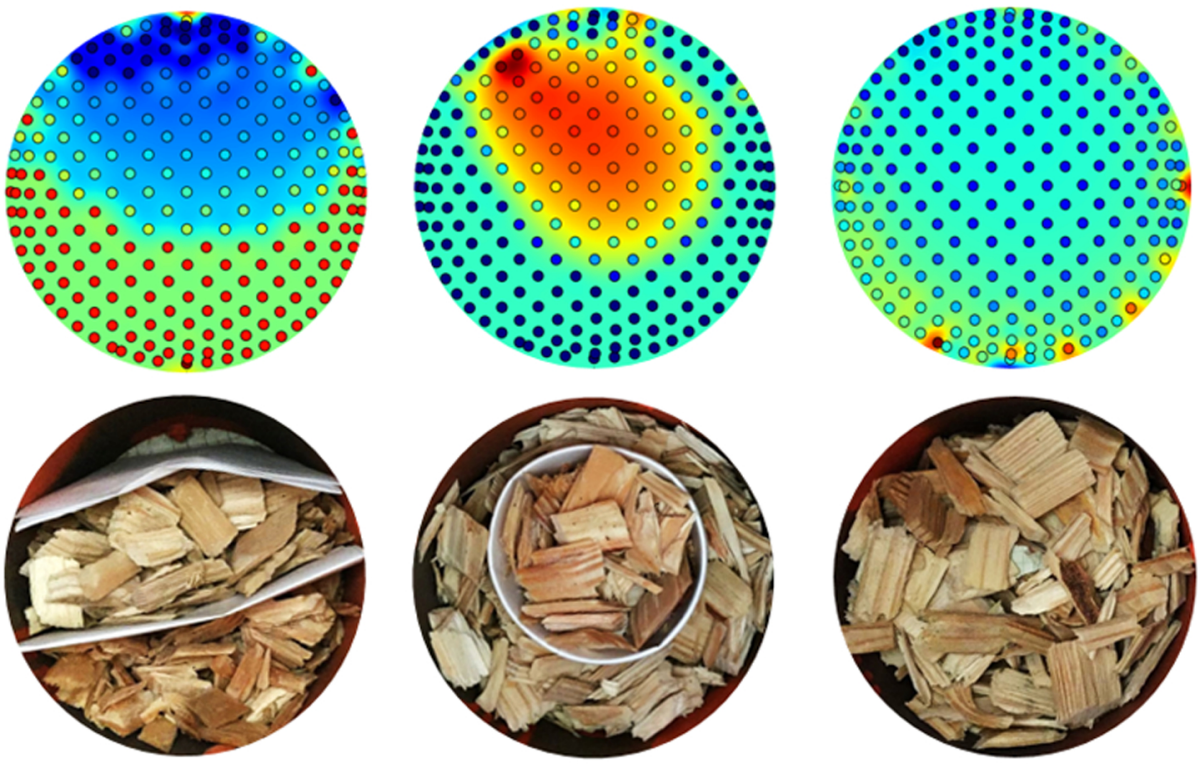
Fig. 4
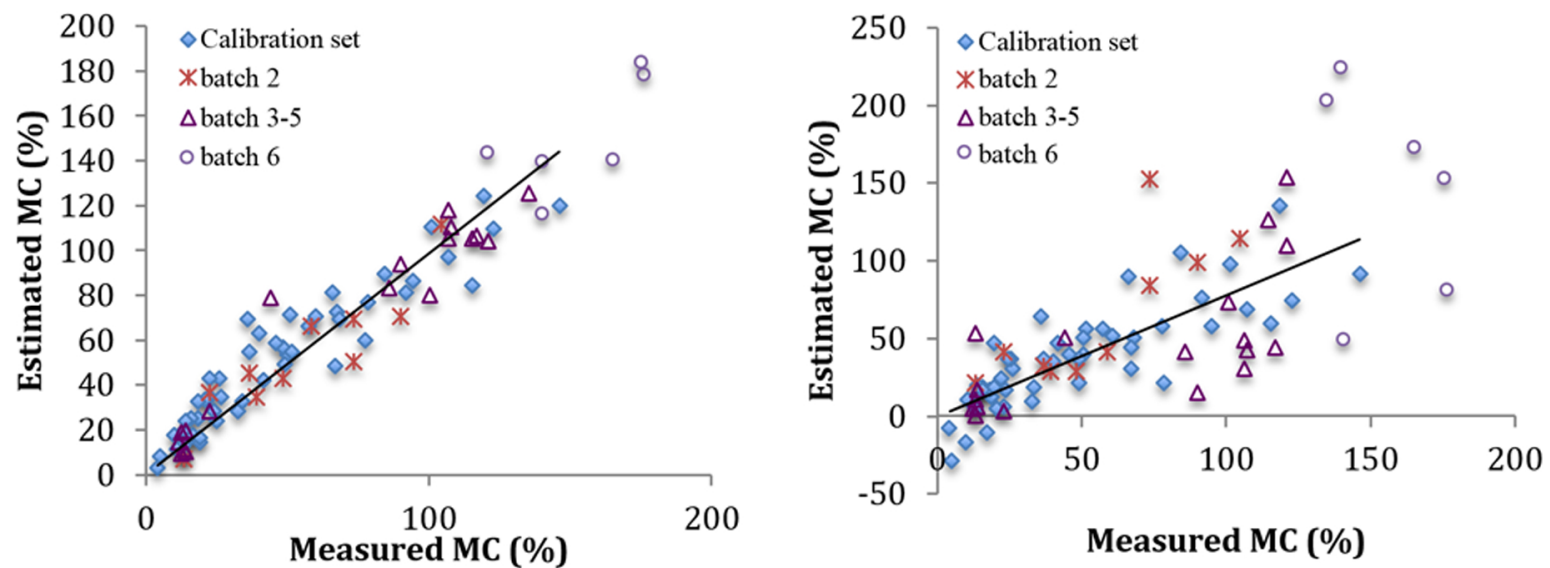

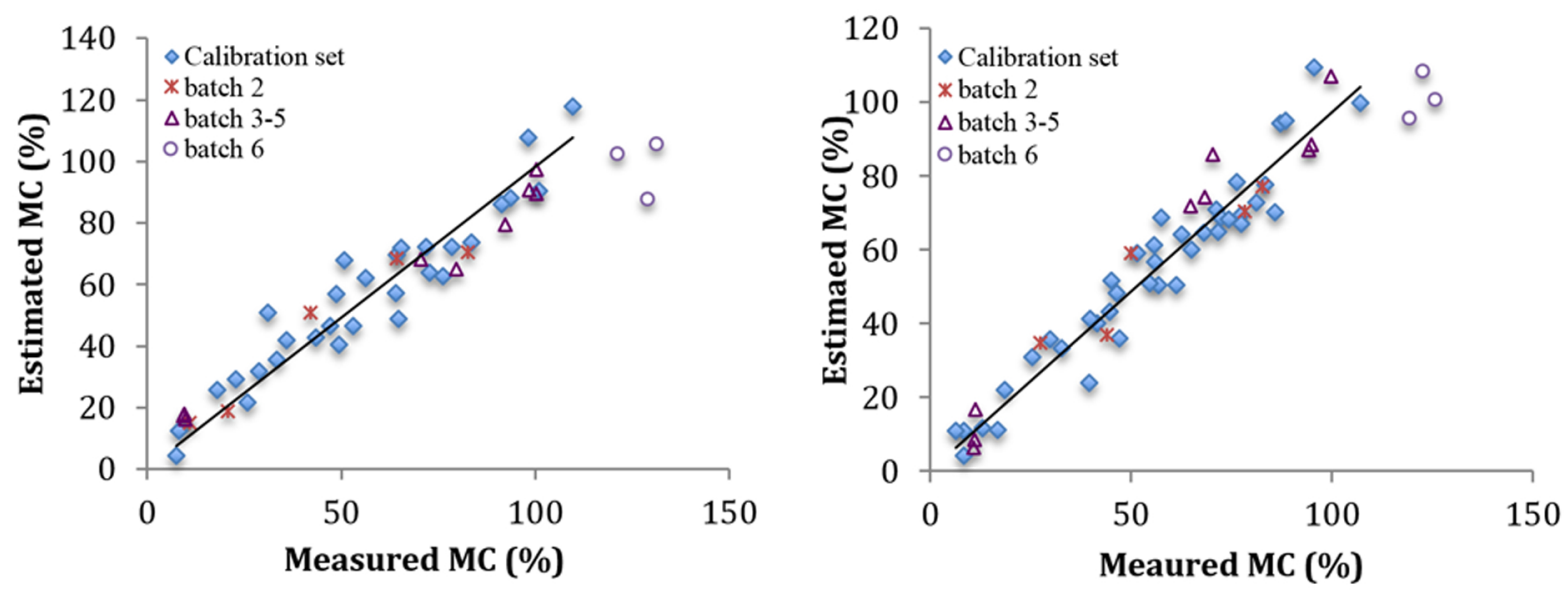
Fig. 6

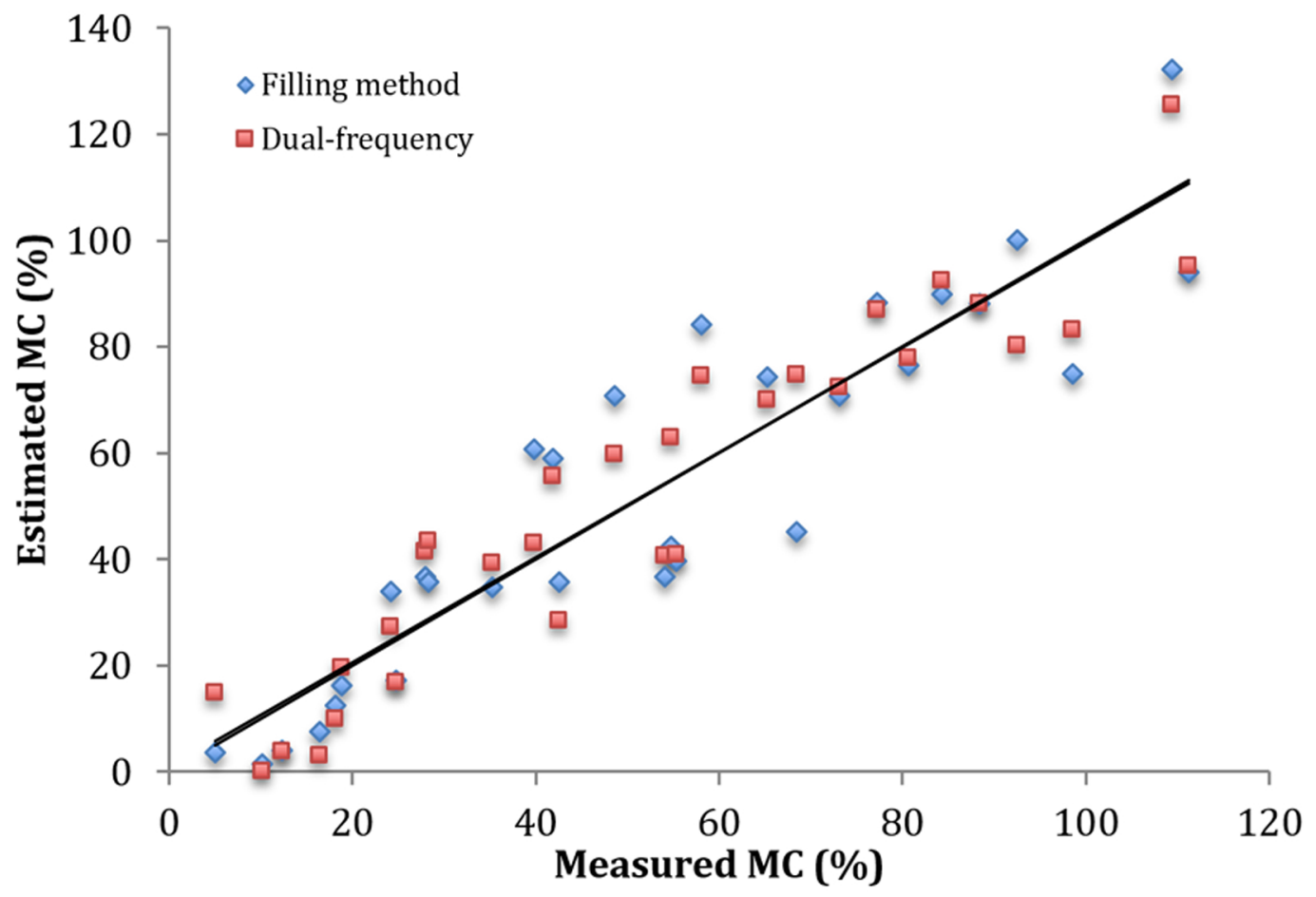


Without modification, however, the ECT sensor tested would not be completely suitable for the accurate measurement of MC in flowing chips. Further work would be required to develop an effective on-line ECT moisture sensor that could effectively deal with simultaneous variation in masses of dry and wet material.

\section{Acknowledgement}

The authors would like to thank Resolute Forest Products of Jackson's Gap, AL and MeadWestvaco of Pittsview, AL, USA for providing the wood chips. We would also like to gratefully acknowledge the support of the Department of Energy [DOE-EE0001036] and US Department of Agriculture - National Institute of Food and Agriculture (USDA-NIFA) in providing funding for the work. 